\title{
Mangosteen pericarp extract inhibits the forma- tion of pentosidine and ameliorates skin elasticity
}

\author{
Rei-ichi Ohno, ${ }^{1}$ Narumi Moroishi, ${ }^{1}$ Hikari Sugawa, ${ }^{1}$ Kazuhiro Maejima, ${ }^{2}$ Musashi Saigusa, ${ }^{2}$ Mikihiro Yamanaka, ${ }^{1,3}$ \\ Mime Nagai, ${ }^{1}$ Morio Yoshimura, ${ }^{4}$ Yoshiaki Amakura ${ }^{4}$ and Ryoji Nagai ${ }^{1}, *$ \\ 'Laboratory of Food and Regulation Biology, Graduate School of Agriculture, Tokai University, Kumamoto 869-1404, Japan \\ ${ }^{2}$ Food Development Laboratories, Nippon Shinyaku Co., Ltd., 14 Nishinosho-monguchi-cho, Kisshoin, Minami-ku, Kyoto 601-8550, Japan \\ ${ }^{3}$ Engineering Department 2, Product Development Center, New Business Development Division, SHARP Corporation, \\ 2613-1 Ichinomoto-cho, Tenri, Nara 632-8567, Japan \\ ${ }^{4}$ College of Pharmaceutical Sciences, Matsuyama University, 4-2 Bunkyo-cho, Matsuyama-shi, Ehime 790-8578, Japan
}

(Received 28 January, 2015; Accepted 12 February, 2015; Published online 16 April, 2015)

\begin{abstract}
The inhibition of advanced glycation end-products (AGEs) by daily meals is believed to become an effective prevention for lifestylerelated diseases. In the present study, the inhibitory effect of hot water extracts of mangosteen (Garcinia mangostana L.) pericarp (WEM) on the formation of pentosidine, one of AGEs, in vitro and in vivo and the remedial effect on skin conditions were measured. WEM significantly inhibited pentosidine formation during gelatin incubation with ribose. Several compounds purified from WEM, such as garcimangosone $D$ and rhodanthenone $B$, were identified as inhibitors of pentosidine formation. Oral administration of WEM at $100 \mathrm{mg} /$ day to volunteer subjects for 3 months reduced the serum pentosidine contents. Because obtaining skin biopsies from healthy volunteers is ethically difficult, AGE accumulation in the skin was estimated by a fluorescence detector. The oral administration of WEM significantly reduced the skin autofluorescence intensity, demonstrating that WEM also reduced AGE accumulation in the skin. Furthermore, the elasticity and moisture content of the skin was also improved by WEM. These results demonstrate that intakes of WEM reduces the glycation stress and results in the improvement of skin conditions.
\end{abstract}

Key Words: AGEs, pentosidine, mangosteen, oxidation, skin elasticity

$G$ arcinia mangostana L. (mangosteen) is cultivated in Southeast Asia, and the fruits have a graceful taste. ${ }^{(1)}$ It is also used in Thailand as a medical plant for the treatment of skin infections and wounds, dysentery, different urinary disorders, cystitis and gonorrhea. ${ }^{(2)}$ The fruit wall contains catechin, procyanidin, anthocyanin and xanthones, such as $\alpha$-mangostin. $\alpha$-Mangostin possess many functions, ${ }^{(3)}$ such as the inhibition of oxidation, ${ }^{(4)}$ inflammation, ${ }^{(5)}$ carcinogenesis and germ growth. ${ }^{(6,7)}$ Juice using the whole fruit, including the peel, ${ }^{(8)}$ and alcohol extracts from the peel are commercially available in the USA. ${ }^{(9)}$ Although water extracts of mangosteen pericarp (WEM) contains few $\alpha$-mangostins, it possesses water soluble polyphenols and shows stronger antioxidative activity than ethanol extracts. ${ }^{(10)}$ However, little is known about the function of water soluble polyphenols of mangosteen.

Amino residues of proteins react with reducing sugars by the Maillard reaction, ${ }^{(11)}$ leading to the formation of early intermediates known as Amadori products, which results in the formation of advanced glycation end-products (AGEs). AGE levels increase with aging, ${ }^{(12-13)}$ diabetic complications, ${ }^{(14-18)}$ and atherosclerosis. ${ }^{(19-22)}$ Among the AGEs structures reported to date, $N^{\varepsilon}$-(carboxymethyl) lysine (CML), a major AGEs antigenic determinant, ${ }^{(23)}$ accumulates in lens crystallins in an age-dependent manner. ${ }^{(24)}$ Pentosidine, one of the fluorescent AGEs structures generated by oxidative conditions, accumulates in the plasma and long-lived tissue proteins, such as the connective and lens tissue proteins collagen and crystallins, respectively. Its level reflects the extent of tissue damage associated with age-related diseases, such as diabetes mellitus, atherosclerosis, and chronic renal failure. ${ }^{(25)}$ The plasma pentosidine levels have also been shown to increase during kidney failure. $^{(26)}$

Glycation of collagen also generates AGEs structures, such as pentosidine, and forms cross-linking between collagen fibers. ${ }^{(25)}$ Accumulation of AGEs in human skin increases with aging and is enhanced by the pathogenesis of diabetes. ${ }^{(27)}$ Antioxidative polyphenols, such as flavonoids, catechin and procyanidin, inhibit the formation of AGEs in vitro. ${ }^{(28-30)}$ Therefore, the inhibitory effect of WEM on the formation of AGEs is predicted because WEM possesses a strong antioxidative activity. ${ }^{(10)}$

We herein determined the inhibitory effects of WEM and its purified compounds on the formation of AGEs in vitro and in vivo. Furthermore, the improvement effect of WEM on the skin elasticity and moisture content were also studied.

\section{Materials and Methods}

Chemicals. Gelatin was purchased from Sigma-Aldrich Japan (Tokyo, Japan). Ribose was purchased from Wako (Osaka, Japan). Horseradish peroxidase (HRP)-conjugated goat anti-mouse IgG antibody was purchased from Kirkegaard Perry Laboratories (Gaithersburg, MD). All other chemicals were of the best grade available from commercial sources.

Preparation of mangosteen pericarp extract. Fresh mangosteen pericarp was boiled in water for $2 \mathrm{~min}$ and then dried at $70^{\circ} \mathrm{C}$ for $8 \mathrm{~h}$. Hot water $(5,600 \mathrm{~L})$ was added to the dried mangosteen pericarp $(600 \mathrm{~kg})$, and the mixture was filtered. The filtrates were concentrated under reduced pressure, 33\% dextrin was added for solid content of extract, and the mixture was then spray-dried to obtain the WEM $(92.1 \mathrm{~kg})$.

Isolation of compounds from mangosteen peel extract. WEM (100 g) was dissolved in water $(500 \mathrm{ml})$, and extracted using $n$-hexane $(1.5 \mathrm{~L})$, ethyl acetate (EtOAc) $(12 \mathrm{~L})$, and $n$ butanol $(\mathrm{BuOH})(12 \mathrm{~L})$, successively, to obtain $n$-hexane $(65.5 \mathrm{mg})$, EtOAc $(2.59 \mathrm{~g})$, and $n$-BuOH $(12.45 \mathrm{~g})$ extracts. The $n-\mathrm{BuOH}$ extract $(7.09 \mathrm{~g})$ was chromatographed over MCI-gel CHP-20P using $\mathrm{MeOH} / \mathrm{H}_{2} \mathrm{O}$ in a stepwise gradient mode. The fractions showing similar HPLC patterns were combined and further purified by column chromatography over Sephadex LH-20, YMC-

*To whom correspondence should be addressed. E-mail: nagai-883@umin.ac.jp 


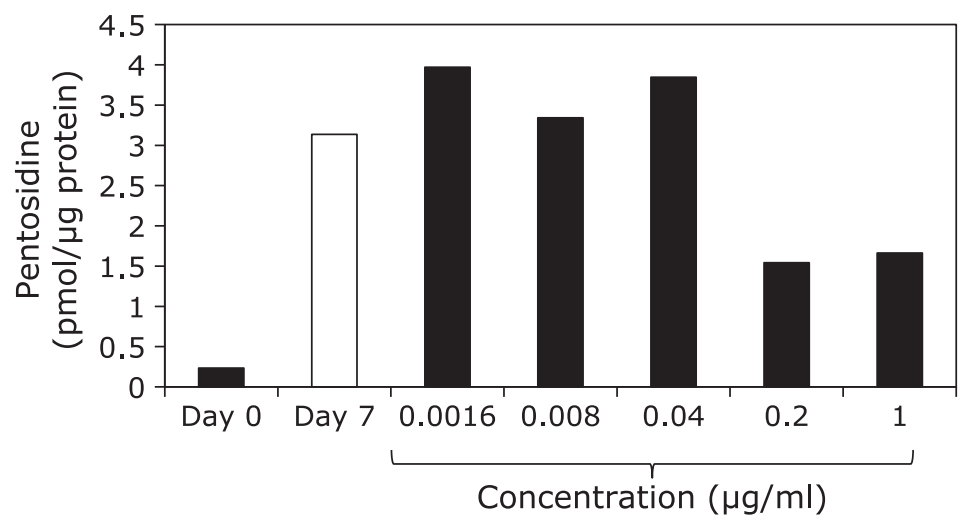

Fig. 1. Inhibitory effects of WEM on pentosidine formation. Gelatin was incubated with ribose in the presence of varying concentrations of WEM extract, and pentosidine contents were measured by HPLC. Pentosidine content was normalized to the protein content of the gelatin.

Garcimangosone D

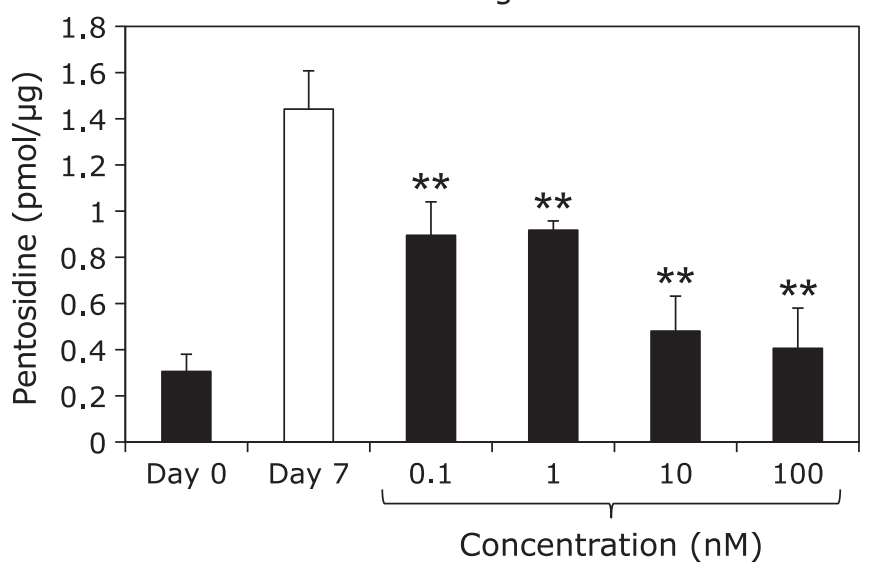

Rhodanthenone B

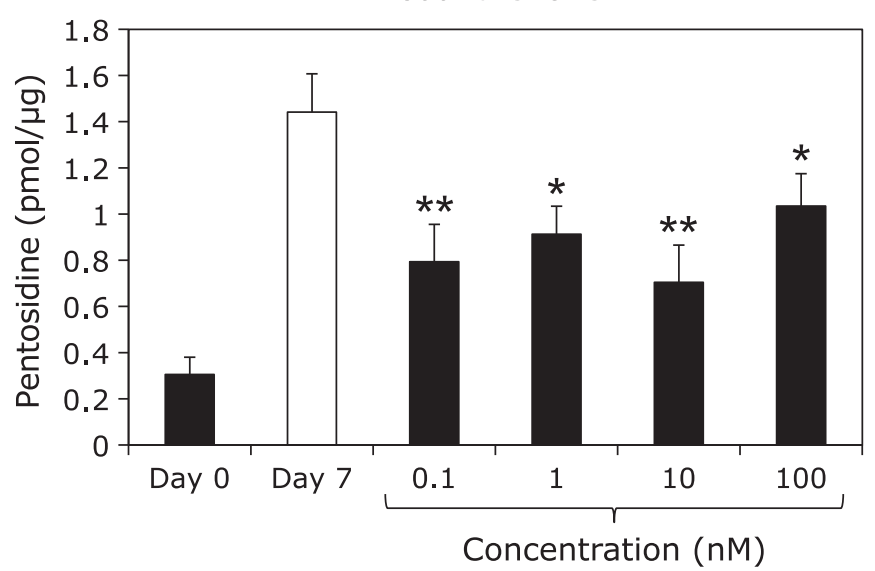

Eucryphin

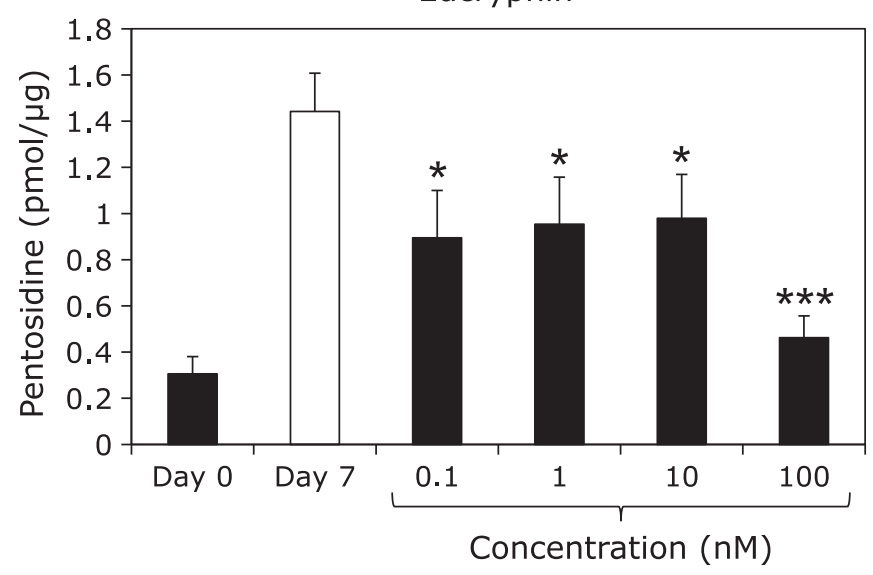

Epicatechin

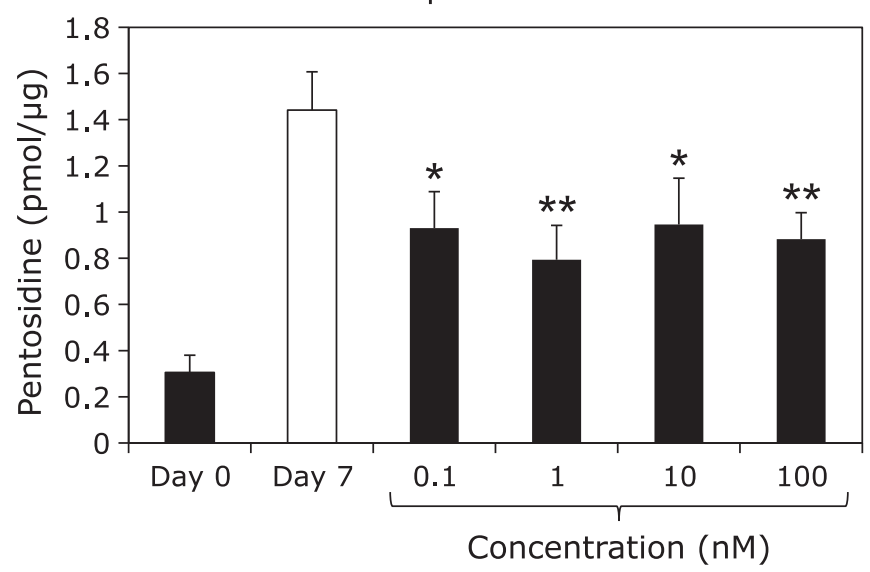

Fig. 2. Inhibitory effects of purified compounds from WEM on pentosidine formation. Gelatin was incubated with ribose in the presence of varying concentrations of WEM extract, and pentosidine contents were measured by HPLC. Pentosidine content was normalized to the protein content of the gelatin. Data are expressed as the mean \pm SD $(n=3)$. ${ }^{*} p<0.05 ; * \star p<0.01 ; * \star * p<0.001$ (with compounds day 7 vs without compounds day 7 ).

gel ODS-AQ, and Chromatorex ODS using aqueous $\mathrm{MeOH}$ to obtain rhodanthenone B (14.0 mg) and epicatechin $(12.4 \mathrm{mg})$. EtOAc $(2.59 \mathrm{~g})$ was purified using column chromatography over Sephadex LH-20, YMC-gel ODS-AQ, and Chromatorex ODS using aqueous $\mathrm{MeOH}$ to obtain epicatechin $(72.5 \mathrm{mg})$, garcimangosone D $(2.2 \mathrm{mg})$, and eucryphin $(2.6 \mathrm{mg})$. These com- pounds were identified by direct comparison with authentic specimens or by comparison of their spectral data with those reported in the literature.

Determination of the inhibitory effect of WEM on pentosidine formation. The compounds were solubilized by dimethyl sulfoxide (Wako). Gelatin $(2 \mathrm{mg} / \mathrm{ml})$ and ribose $(30 \mathrm{mM})$ were 
incubated with WEM $(0.0016-1 \mu \mathrm{g} / \mathrm{ml})$ or purified compounds $(0.1-100 \mathrm{nM})$ in $200 \mathrm{mM}$ sodium phosphate buffer $(\mathrm{pH} 7.2)$ at $37^{\circ} \mathrm{C}$ for 7 days. The samples was dialyzed against PBS at $4^{\circ} \mathrm{C}$ for $18 \mathrm{~h}$, and the protein concentration was measured by the bicinchoninic acid protein assay (Thermo Scientific K.K., Japan), followed by the determination of pentosidine by high performance liquid chromatography (HPLC) analysis as described below.

Determination of the pentosidine content by HPLC.

A pentosidine standard was prepared as described previously, ${ }^{(16)}$ and pentosidine was measured as described previously. ${ }^{(32)}$ Briefly, ribose-gelatin samples $(50 \mu \mathrm{g}$ each) were dried in vacuo and hydrolyzed by $6 \mathrm{~N}$ hydrochloric acid $(\mathrm{HCl})$ at $110^{\circ} \mathrm{C}$ for $24 \mathrm{~h}$ in sealed test tubes. Serum samples $(0.1 \mathrm{ml}$ each $)$ were mixed with an equal volume of $12 \mathrm{~N} \mathrm{HCl}(6 \mathrm{~N}$, final concentration) and hydrolyzed at $110^{\circ} \mathrm{C}$ for $24 \mathrm{~h}$ in sealed test tubes. The $\mathrm{HCl}$ was dried in vacuo and solubilized again with $0.5 \mathrm{ml}$ of distilled water. The samples were applied to cation exchange columns (Strata-X-C Polymeric Strong Cation, $30 \mathrm{mg} / \mathrm{ml}$ ) (Phenomenex, California), eluted by $7 \%$ ammonia (Nacalai Tesque, Kyoto, Japan) and dried in vacuo. The samples were resolubilized in $0.3 \mathrm{ml}$ of $16 \%$ acetonitrile solution, followed by pentosidine analysis via HPLC. ${ }^{(32)}$

Estimation of skin AGEs by autofluorescence. The inhibitory effect of WEM on AGE accumulation in the skin was estimated by measuring fingertip autofluorescence because it is difficult to obtain skin samples from healthy subjects. Similar to previous reports, ${ }^{(33)}$ skin autofluorescence was recorded with a prototype consisting of a light emitting diode (LED) light source, a spectral apparatus system by a 2048-pixel CCD linear image sensor and grating and a biantennary silica-based optical fiber. A skin surface area approximately $0.38 \mathrm{~mm}$ in diameter was irradiated by an LED light source of $365 \mathrm{~nm}$ at excitation peak. For the detection of fluorescence signals at the same location on the skin, a Y-shaped flexible optical fiber coated in polyurethane was used. The quartz $\mathrm{Y}$ shape fiber consists of two core fibers $(0.6 \mathrm{~mm}$ in diameter), and one side of the fibers was connected to a spectrometer while the other side was connected to an LED light source. The skin autofluorescence spectrum showed a stable emission peak at 450-460 $\mathrm{nm}$. In the previous studies, skin autofluorescence measurements were performed on the volar side of the lower arm. All measurements were performed in a semi-dark, temperature-controlled room.

Administration of WEM to volunteer subjects. The study was conducted in accordance with the Declaration of Helsinki as a statement of ethical principles for medical research involving human subjects, including research on identifiable human material and data. The experimental protocol was approved by the ethics review committee of Kitahorie Hospital for intervention trials, and AGE analysis was approved by the ethics review committee of Tokai University (\#14036). This study was conducted as an open trial which recruited healthy women between the ages of 32 to 48 years old $(n=11)$. All subjects provided written informed consent. The median fasting blood glucose and hemoglobin Alc of the subjects were $83.6 \pm 4.6 \mathrm{mg} / \mathrm{dl}$ and $5.25 \pm 0.21$, respectively. Two tablets (100 mg WEM in total), once a day, were administered to each subject and continued for 12 weeks. Routine blood and skin function testing were evaluated every 4 weeks.

Measurement of the elasticity and moisture content of the skin. Measurement of the skin moisture and elasticity were conducted at $25^{\circ} \mathrm{C}$ after $30 \mathrm{~min}$ of rest. Skin elasticity was measured by a skin viscoelasticity analyzer, Cutometer (MPA580; Courage \& Khazaka, Kern, Garmany). ${ }^{(34)}$ The skin surface of left upper arm was drawn into the aperture of the probe by negative pressure, and the penetration depth of the skin was measured using prisms. The skin elasticity on the internal lesion of the left upper arm was analyzed, and 2 skin elasticity indices were measured: the recovery ratio of the skin length (RR) and the ratio of elasticity during constriction (RE). The measurement of moisture contents on the face was conducted by the Robo Skin Analyzer (RSA50;
Inforward, Inc., Tokyo, Japan). ${ }^{(35)}$

Statistical analysis. All data were expressed as the mean \pm SD. Differences between the groups were examined using the Mann-Whitney $U$ test and the non-repeated measures ANOVA. A $p$ value of less than 0.05 was considered to be statistically significant.

\section{Results}

Inhibitory effects of WEM extract and purified compounds on pentosidine formation. Gelatin was incubated with ribose in the presence of varying concentrations of WEM extract, and the pentosidine contents were measured by HPLC. As shown in Fig. $1,0.2 \mu \mathrm{g} / \mathrm{ml}$ of WEM showed $\sim 51 \%$ inhibition of pentosidine formation. Therefore, compounds that inhibit pentosidine formation were isolated from WEM. Several purified compounds from $0.1 \mathrm{nM}$ WEM, such as garcimangosone $\mathrm{D}$, eucryphin, rhodanthenone B and epicatechin, inhibited pentosidine formation by $38 \%, 38 \%, 45 \%$ and $35 \%$, respectively (Fig. 2). Garcimangosone D in particular showed the highest inhibitory effect among the purified compounds. These results demonstrate that WEM possesses several compounds that inhibit pentosidine
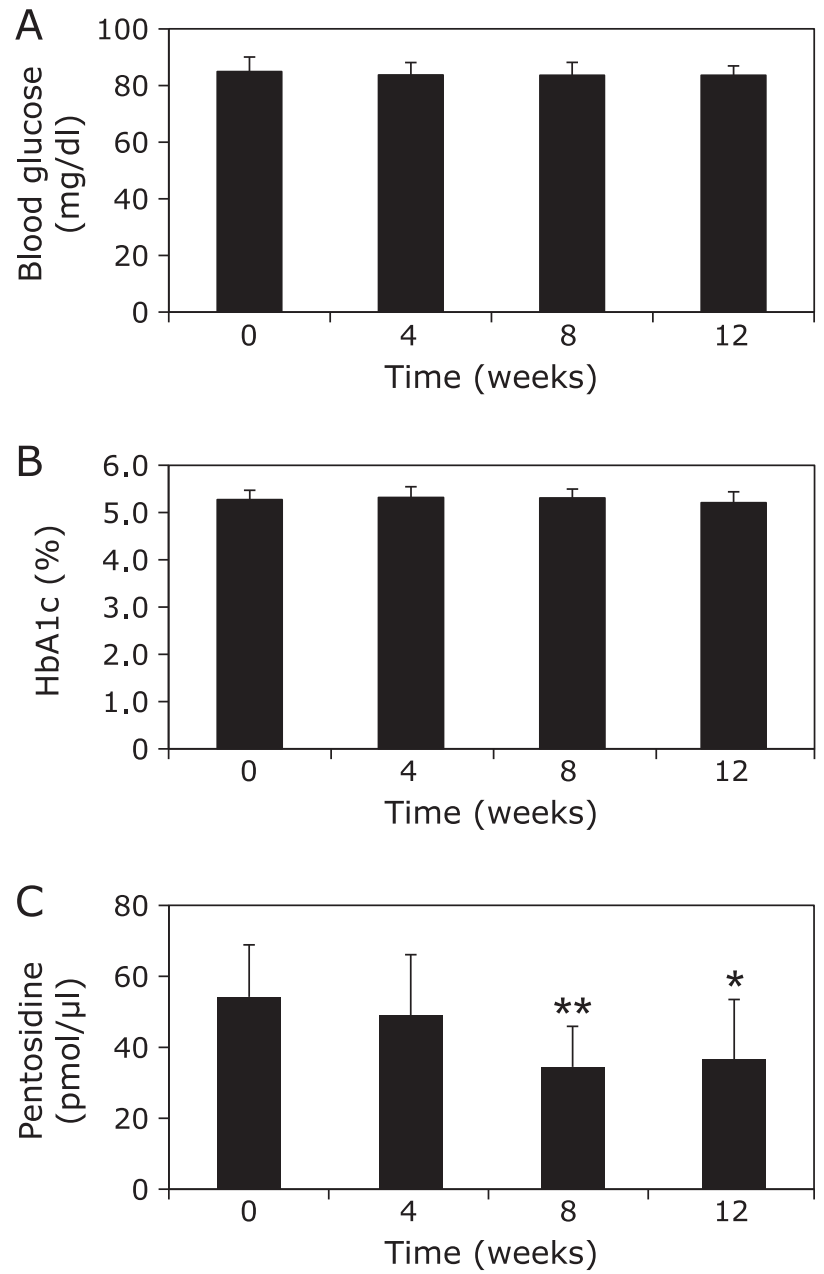

Fig. 3. Effects of WEM on biochemical parameters and pentosidine formation in vivo. WEM was administered to healthy subjects, and the serum pentosidine levels were determined by HPLC. Two tablets (100 mg WEM in total) were administered once a day to healthy women $(n=11)$ for 12 weeks. The blood glucose (A), HbA1c (B), and pentosidine (C) levels were evaluated every 4 weeks. ${ }^{*} p<0.05 ;{ }^{*} p<0.01$ (vs before WEM administration). 
A
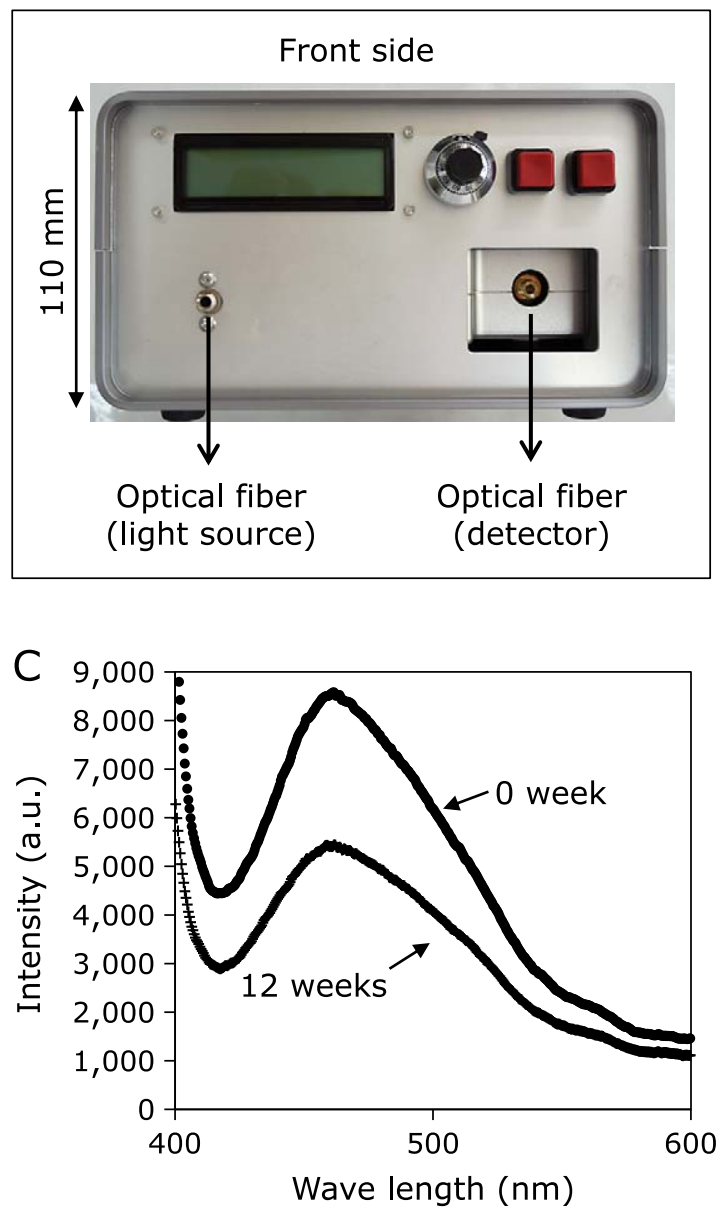

B
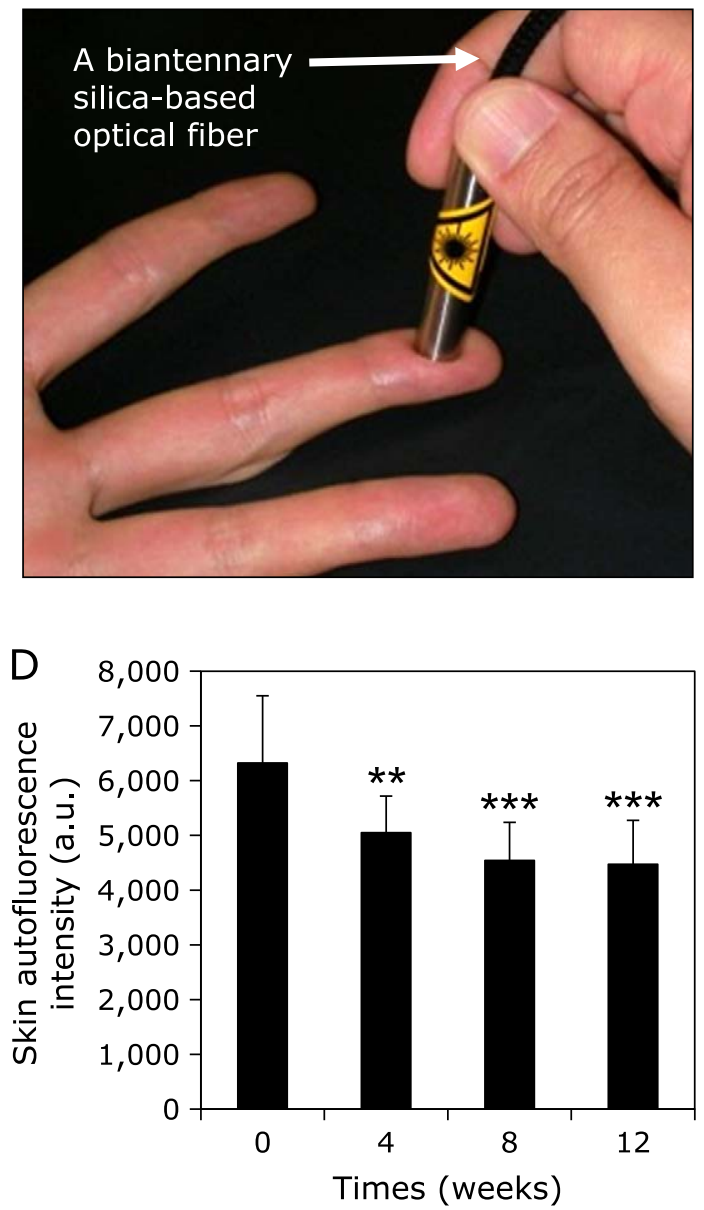

Fig. 4. The inhibitory effect of WEM on AGE accumulation in the skin. Skin autofluorescence was recorded with a prototype consisting of a LED light source (A), a spectral apparatus system by a 2048-pixel CCD linear image sensor and grating and a biantennary silica-based optical fiber (B). A skin surface area was irradiated by an LED light source of $365 \mathrm{~nm}$ at excitation peak, and the skin autofluorescence spectrum showed a stable emission peak at $450-460 \mathrm{~nm}$ (C). The inhibitory effect of WEM on AGE accumulation in the skin was estimated by measuring fingertip autofluorescence $(n=11)(D) .{ }^{* *} p<0.01 ; * * * p<0.001$ (vs before WEM administration).

formation. Therefore, WEM was administered to healthy subjects, and the serum pentosidine level was determined by HPLC. Although the fasting blood glucose (Fig. 3A) and HbAlc (Fig. 3B) levels did not change in the subjects who received $100 \mathrm{mg}$ /day $\mathrm{WEM}$, the serum pentosidine levels $(54.4 \pm 15.4 \mathrm{pmol} / \mu \mathrm{l})$ were slightly decreased at 4 weeks and significantly decreased at 8 weeks ( $p<0.005$ vs before WEM administration) then reached a plateau level at 12 weeks ( $p<0.05$ vs before WEM administration) (Fig. 3C). Furthermore, AGEs accumulation in the skin was estimated by measuring the skin fluorescence (Fig. 4A and B). A typical spectrum at wave length points of skin autofluorescence was compared initial results to that after 12 weeks. Each spectrum was acquired from skin surface area that was irradiated by an LED light source of $365 \mathrm{~nm}$ at excitation peak, and the skin autofluorescence spectrum showed a stable emission peak at 450 $460 \mathrm{~nm}$ (Fig. 4C). The fluorescence on the fingertips $(n=11)$ was significantly decreased in the subjects after receiving $100 \mathrm{mg}$ /day WEM in a time-dependent manner and reached a plateau level at 12 weeks (Fig. 4D).

Effect of WEM on the elasticity and moisture content of the skin. Although the RR of the subjects was 0.865 , it was significantly improved at 4 weeks $(0.929 ; p<0.005$ vs before WEM administration) and the elasticity of the skin remained constant during the 12 weeks (Fig. 5A). A similar tendency was observed with the RE. The RE of the subjects significantly improved at 4 weeks $(0.663$ vs $0.715 ; p<0.01$ vs before WEM administration) and was maintained during the 12 weeks (Fig. 5B).

Furthermore, the average moisture content of the skin in the subjects before WEM administration (72.8) increased in a timedependent manner and became 84.1 at 12 weeks $(p<0.001$ vs before WEM administration) (Fig. 5C). These results strongly demonstrated that oral administration of WEM improved the skin conditions, such as elasticity and moisture content, in the subjects at 4 weeks.

\section{Discussion}

WEM possesses several functions, such as the scavenging activity of the free radical DPPH and neuroprotective activity by inhibiting oxidative stress in vitro. ${ }^{(10,36)}$ These studies suggest the possibility that water-soluble polyphenols in WEM may inhibit oxidation, resulting in the inhibition of pentosidine formation in vivo.

Pentosidine formation was inhibited by $0.2 \mu \mathrm{g} / \mathrm{ml}$ of WEM (Fig. 1) and $0.1-100 \mathrm{nM}$ of purified compounds from WEM (Fig. 2). Previous studies demonstrated that monomeric flavonoids inhibited pentosidine formation at concentrations of 25 $250 \mu \mathrm{M},{ }^{(28)}$ suggesting that WEM contains strong compounds 

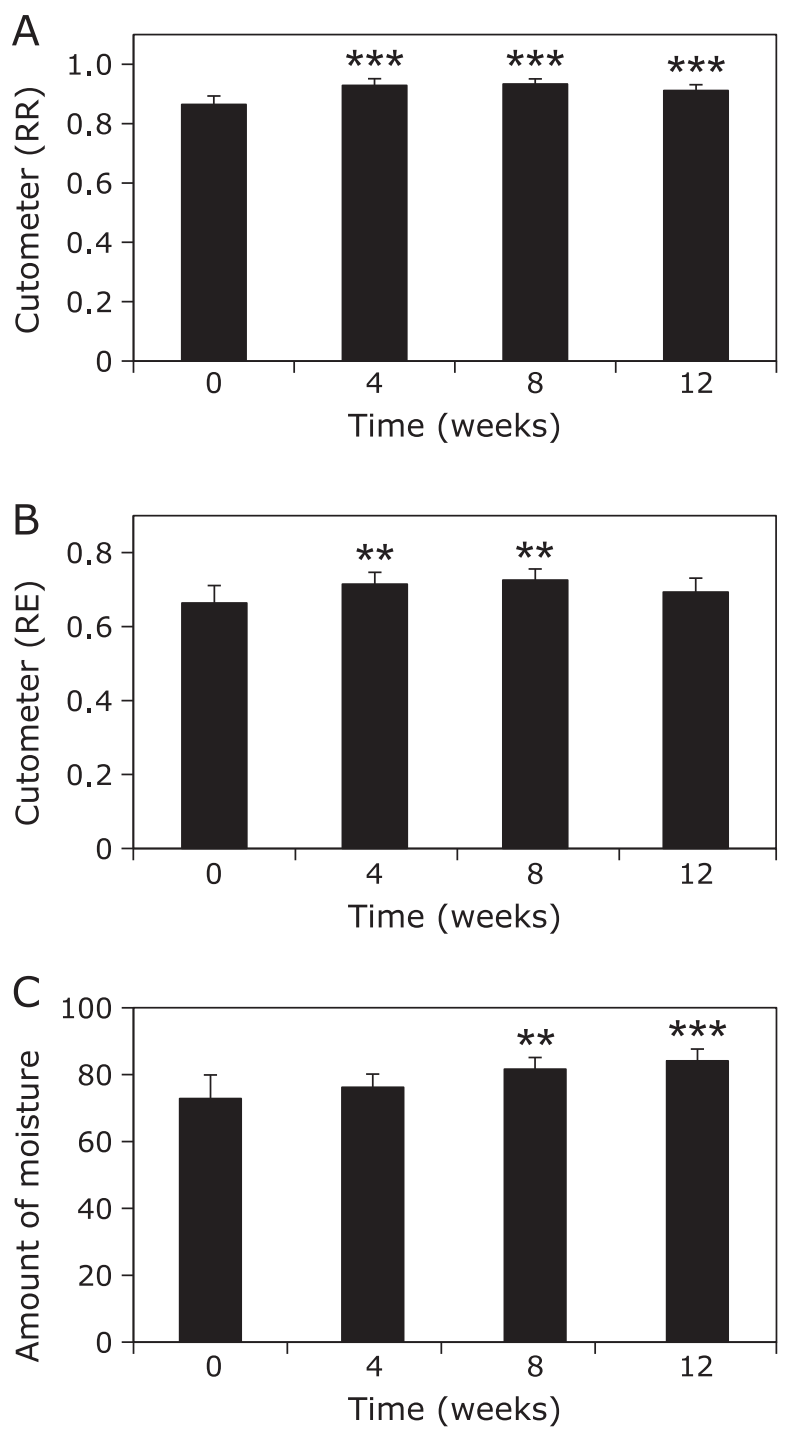

Fig. 5. Effect of WEM on the elasticity and moisture content of the skin. Skin elasticity was measured by a skin viscoelasticity analyzer. Skin elasticity on the internal lesion of left upper arm was analyzed and 2 skin elasticity indices were measured: $(A)$ the recovery ratio of the skin length (RR), and (B) the ratio of elasticity during constriction (RE). The measurement of moisture contents on the face $(C)$ was conducted by a Robo Skin Analyzer. The inhibitory effect of WEM on AGE accumulation in the skin was estimated by measuring fingertip autofluorescence (D). ${ }^{* *} p<0.01 ;{ }^{* *} p<0.001$ (vs before WEM administration).

capable of suppressing pentosidine formation in vitro. Moreover, a human intervention study showed that the oral administration of WEM reduced the serum pentosidine level in subjects (Fig. 3A). This study provides the first evidence that natural compounds reduced pentosidine formation not only in vitro but also in vivo.

Several compounds purified from WEM, such as garcimangosone $\mathrm{D}$, eucryphin, rhodanthenone $\mathrm{B}$ and epicatechin, were observed to play an important role in the inhibition of pentosidine formation (Fig. 2). In particular, benzophenone compounds, i.e., garcimangosone $\mathrm{D}$ and rhodanthenone $\mathrm{B}$, showed stronger inhibitory effects on pentosidine formation among the purified compounds. Although rhodanthenone B was originally isolated from Gentiana rhodantha Franch. (gentianaceae), ${ }^{(37)}$ there are no previous report on the function and the presence of this compound. We observed that rhodanthenone $\mathrm{B}$ and garcimangosone $\mathrm{D}$ were $0.08 \%$ and less than $0.01 \%$, respectively (data not shown) of the WEM, thus, rhodanthenone B may play an important role in the inhibition of pentosidine formation. These data demonstrate that daily WEM intake may be effective in inhibiting pentosidine compared with other natural products.

Because obtaining skin biopsies from healthy volunteers is ethically difficult, AGE accumulation in the skin was estimated by a fluorescence detector. Dong et $a l^{\left({ }^{(38)}\right.}$ previously demonstrated that fluorescence intensity correlated with the pentosidine contents in the bone, and Couppé et al ${ }^{(39)}$ also reported that skin fluorescence intensity correlated with the pentosidine levels in the patellar tendon, demonstrating that skin autofluorescence is highly correlated with the accumulation of AGEs in vivo. In the present study, the oral administration of WEM significantly reduced the plasma pentosidine levels (Fig. 3C) and the skin autofluorescence intensity (Fig. 4D), strongly demonstrating that WEM also reduced AGE accumulation in the skin. The fluorescent wavelength of pentosidine is excitation at $335 \mathrm{~nm}$ and emission at $385 \mathrm{~nm}$, whereas skin fluorescence was measured by excitation at $365 \mathrm{~nm}$ and emission at $440 \mathrm{~nm}$, suggesting that administration of WEM reduced several AGEs in vivo.

Collagen and elastin molecules are involved in skin elasticity, which was reduced in accordance with aging. Lysine and arginine on collagen molecules are modified by AGEs and result in the denaturation of the three-dimensional structure of skeletal proteins and enzymes. Pentosidine is known as one of the cross-linked AGE structures and generates unfavorable cross-linking between collagen molecules. ${ }^{(4)}$ Oral administration of WEM to the subjects resulted in reduced serum pentosidine levels (Fig. 3C) and skin AGE-fluorescence (Fig. 4D) and ameliorated the skin elasticity and moisture contents on the face (Fig. 5). Our study demonstrates that oral administration of WEM inhibits AGE formation and results in improved skin conditions in vivo. Saito et al. ${ }^{(40)}$ demonstrated that the bone quality correlated with the bone pentosidine concentration. Furthermore, Arai et al. ${ }^{(41)}$ reported that the levels of pentosidine in the blood are elevated in patients with schizophrenia, and the association of AGEs and mental illness is currently being investigated. Therefore, AGE inhibitors have been developed for the prevention of lifestyle-related diseases and psychiatric diseases. ${ }^{(42)}$ Because the daily intake of a WEM tablet $(100 \mathrm{mg})$ does not lead to serious side effects, intake WEM should be considered as a new food for preventing the accumulation of AGEs and improving functional disorders due to aging.

\section{Acknowledgments}

This work was supported in part by Grants-in-Aid for Scientific Research (No. 24300260 and 24650482 to Ryoji Nagai) from the Ministry of Education, Science, Sports and Culture of Japan. This work was also supported in part by grants from the Research Institute of Agriculture of Tokai University.

\section{References}

1 Obolskiy D, Pischel I, Siriwatanametanon N, Heinrich M. Garcinia mangostana L.: a phytochemical and pharmacological review. Phytother Res 2009; 23: 1047-1065.

2 Nakatani K, Nakahata N, Arakawa T, Yasuda H, Ohizumi Y. Inhibition of cyclooxygenase and prostaglandin E2 synthesis by gamma-mangostin, a xanthone derivative in mangosteen, in C6 rat glioma cells. Biochem Pharmacol

2002; 63: 73-79.

3 Kondo M, Zhang L, Ji H, Kou Y, Ou B. Bioavailability and antioxidant effects of a xanthone-rich Mangosteen (Garcinia mangostana) product in humans. J Agric Food Chem 2009; 57: 8788-8792.

4 Moongkarndi P, Jaisupa N, Samer J, et al. Comparison of the biological activity of two different isolates from mangosteen. J Pharm Pharmacol 2014; 
66: 1171-1179.

5 Chen LG, Yang LL, Wang CC. Anti-inflammatory activity of mangostins from Garcinia mangostana. Food Chem Toxicol 2008; 46: 688-693.

6 Akao Y, Nakagawa Y, Iinuma M, Nozawa Y. Anti-cancer effects of xanthones from pericarps of mangosteen. Int J Mol Sci 2008; 9: 355-370.

7 Kaomongkolgit R, Jamdee K, Chaisomboon N. Antifungal activity of alphamangostin against Candida albicans. J Oral Sci 2009; 51: 401-406.

8 Udani JK, Singh BB, Barrett ML, Singh VJ. Evaluation of Mangosteen juice blend on biomarkers of inflammation in obese subjects: a pilot, dose finding study. Nutr J 2009; 8: 48.

9 Balunas MJ, Su B, Brueggemeier RW, Kinghorn AD. Xanthones from the botanical dietary supplement mangosteen (Garcinia mangostana) with aromatase inhibitory activity. J Nat Prod 2008; 71: 1161-1166.

10 Ngawhirunpat T, Opanasopi P, Sukma M, Sittisombut C, Kat A, Adachi I. Antioxidant, free radical-scavenging activity and cytotoxicity of different solvent extracts and their phenolic constituents from the fruit hull of mangosteen (Garcinia mangostana). Pharm Biol 2010; 48: 55-62.

11 Nagai R, Matsumoto K, Ling X, Suzuki H, Araki T, Horiuchi S. Glycolaldehyde, a reactive intermediate for advanced glycation end products, plays an important role in the generation of an active ligand for the macrophage scavenger receptor. Diabetes 2000; 49: 1714-1723.

12 Araki N, Ueno N, Chakrabarti B, Morino Y, Horiuchi S. Immunochemical evidence for the presence of advanced glycation end products in human lens proteins and its positive correlation with aging. J Biol Chem 1992; 267: 10211-10214.

13 Kimura T, Takamatsu J, Ikeda K, Kondo A, Miyakawa T, Horiuchi S. Accumulation of advanced glycation end products of the Maillard reaction with age in human hippocampal neurons. Neurosci Lett 1996; 208: 53-56.

14 Makino H, Shikata K, Hironaka K, et al. Ultrastructure of nonenzymatically glycated mesangial matrix in diabetic nephropathy. Kidney Int 1995; 48: 517526.

15 Yamada K, Miyahara Y, Hamaguchi K, et al. Immunohistochemical study of human advanced glycosylation end-products (AGE) in chronic renal failure. Clin Nephrol 1994; 42: 354-361.

16 Imai N, Nishi S, Suzuki Y, et al. Histological localization of advanced glycosylation end products in the progression of diabetic nephropathy. Nephron 1997; 76: 153-160.

17 Hammes H-P, Weiss A, Hess S, et al. Modification of vitronectin by advanced glycation alters functional properties in vitro and in the diabetic retina. Lab Invest 1996; 75: 325-338.

18 Murata T, Nagai R, Ishibashi T, Inomuta H, Ikeda K, Horiuchi S. The relationship between accumulation of advanced glycation end products and expression of vascular endothelial growth factor in human diabetic retinas. Diabetologia 1997; 40: 764-769.

19 Kume S, Takeya M, Mori T, et al. Immunohistochemical and ultrastructural detection of advanced glycation end products in atherosclerotic lesions of human aorta with a novel specific monoclonal antibody. Am J Pathol 1995; 147: 654-667.

20 Nakamura Y, Horii Y, Nishino T, et al. Immunohistochemical localization of advanced glycosylation endproducts in coronary atheroma and cardiac tissue in diabetes mellitus. Am J Pathol 1993; 143: 1649-1656.

21 Meng J, Sakata N, Takebayashi S, et al. Advanced glycation end products of the Maillard reaction in aortic pepsin-insoluble and pepsin-soluble collagen from diabetic rats. Diabetes 1996; 45: 1037-1043.

22 Nagai R, Hayashi CM, Xia L, Takeya M, Horiuchi S. Identification in human atherosclerotic lesions of GA-pyridine, a novel structure derived from glycolaldehyde-modified proteins. J Biol Chem 2002; 277: 48905-48912.

23 Ahmed MU, Thorpe SR, Baynes JW. Identification of $\mathrm{N}^{\varepsilon}$-carboxymethyllysine as a degradation product of fructoselysine in glycated protein. $J$ Biol
Chem 1986; 261: 4889-4894.

24 Dunn JA, Patrick JS, Thorpe SR, Baynes JW. Oxidation of glycated proteins: age-dependent accumulation of $\mathrm{N}^{\varepsilon}$-(carboxymethyl)lysine in lens proteins. Biochemistry 1989; 28: 9464-9468.

25 Sell DR, Monnier VM. Structure elucidation of a senescence cross-link from human extracellular matrix. Implication of pentoses in the aging process. $J$ Biol Chem 1989; 264: 21597-21602.

26 Miyata T, Ueda Y, Shinzato T, et al. Accumulation of albumin-linked and free-form pentosidine in the circulation of uremic patients with end-stage renal failure: renal implications in the pathophysiology of pentosidine. $\mathrm{J} \mathrm{Am}$ Soc Nephrol 1996; 7: 1198-1206.

27 Dunn JA, McCance DR, Thorpe SR, Lyons TJ, Baynes JW. Age-dependent accumulation of $\mathrm{N}$ epsilon-(carboxymethyl)lysine and $\mathrm{N}$ epsilon-(carboxymethyl)hydroxylysine in human skin collagen. Biochemistry 1991; 30: 12051210.

28 Urios P, Grigorova-Borsos AM, Sternberg M. Flavonoids inhibit the formation of the cross-linking AGE pentosidine in collagen incubated with glucose, according to their structure. Eur J Nutr 2007; 46: 139-146.

29 Yokozawa T, Nakagawa T. Inhibitory effects of Luobuma tea and its components against glucose-mediated protein damage. Food Chem Toxicol 2004; 42: 975-981.

30 Cai Q, Li BY, Gao HQ, et al. Grape seed procyanidin b2 inhibits human aortic smooth muscle cell proliferation and migration induced by advanced glycation end products. Biosci Biotechnol Biochem 2011; 75: 1692-1697.

31 Motomura K, Fujiwara Y, Kiyota N, et al. Astragalosides isolated from the root of astragalus radix inhibit the formation of advanced glycation end products. J Agric Food Chem 2009; 57: 7666-7672.

32 Nakano M, Kubota M, Owada S, Nagai R. The pentosidine concentration in human blood specimens is affected by heating. Amino Acids $2013 ; \mathbf{4 4}$ : $1451-$ 1456.

33 Meerwaldt R, Graaff R, Oomen PH. Simple non-invasive assessment of advanced glycation endproduct accumulation. Diabetologia 2004; 47: 13241330.

34 Akhtar N, Zaman SU, Khan BA, Amir MN, Ebrahimzadeh MA. Calendula extract: effects on mechanical parameters of human skin. Acta Pol Pharm 2011; 68: 693-701.

35 Kakudo N, Kushida S, Suzuki K, Kusumoto K. Effects of glycolic acid chemical peeling on facial pigment deposition: evaluation using novel computer analysis of digital-camera-captured images. $J$ Cosmet Dermatol 2013; 12: 281-286.

36 Fu C, Loo AE, Chia FP, Huang D. Oligomeric proanthocyanidins from mangosteen pericarps. J Agric Food Chem 2007; 55: 7689-7694.

37 Xu M, Zhang M, Wang D, Yang CR, Zhang YJ. Phenolic compounds from the whole plants of Gentiana rhodantha (Gentianaceae). Chem Biodivers 2011; 8: 1891-1900.

38 Dong XN, Qin A, Xu J, Wang X. In situ accumulation of advanced glycation endproducts (AGEs) in bone matrix and its correlation with osteoclastic bone resorption. Bone 2011; 49: 174-183.

39 Couppé C, Svensson RB, Grosset JF, et al. Life-long endurance running is associated with reduced glycation and mechanical stress in connective tissue. Age (Dordr) 2014; 36: 9665.

40 Saito M, Marumo K. Collagen cross-links as a determinant of bone quality: a possible explanation for bone fragility in aging, osteoporosis, and diabetes mellitus. Osteoporos Int 2010; 21: 195-214.

41 Arai M, Yuzawa H, Nohara I, et al. Enhanced carbonyl stress in a subpopulation of schizophrenia. Arch Gen Psychiatry 2010; 67: 589-597.

42 Nagai R, Shirakawa J, Ohno R, Moroishi N, Nagai M. Inhibition of AGEs formation by natural products. Amino Acids 2014; 46: 261-266. 